\title{
BMJ Open Positive lifestyle changes around the time of pregnancy: a cross-sectional study
}

\author{
Linda M O'Keeffe, ${ }^{1,2}$ Darren L Dahly, ${ }^{2,3}$ Marion Murphy, ${ }^{2,4}$ Richard A Greene, ${ }^{5}$ \\ Janas M Harrington, ${ }^{2}$ Paul Corcoran, ${ }^{2,5}$ Patricia M Kearney ${ }^{2}$
}

To cite: O'Keeffe LM, Dahly DL, Murphy M, et al. Positive lifestyle changes around the time of pregnancy: a cross-sectional study. BMJ Open 2016;6: e010233. doi:10.1136/ bmjopen-2015-010233

- Prepublication history for this paper is available online. To view these files please visit the journal online (http://dx.doi.org/10.1136/ bmjopen-2015-010233).

Received 9 October 2015 Revised 15 December 2015 Accepted 12 January 2016

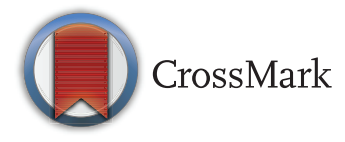

For numbered affiliations see end of article.

Correspondence to Dr Linda M O'Keeffe; Linda.okeeffe@bristol.ac.uk

\section{ABSTRACT}

Objectives: To examine the prevalence of positive lifestyle behaviours before and during pregnancy in Ireland.

Design: Cross-sectional study.

Setting: Population-based study in Ireland.

Participants: A total of 718 women of predominantly Caucasian origin from the Pregnancy Risk Assessment Monitoring System (PRAMS), Ireland, were included.

Primary and secondary outcome measures:

Positive lifestyle behaviour changes before and during pregnancy in Ireland on alcohol consumption, smoking, folate use and nutrition.

Results: Of 1212 women surveyed, 718 (59\%) responded. $26 \%$ were adherent to all three recommendations on alcohol consumption, smoking and folate use before pregnancy. This increased to $39 \%$ for the same three behaviours during pregnancy, with greater increases in adherence observed among women with the lowest adherence before pregnancy. Age, education and ethnicity gaps in adherence before pregnancy appeared to narrow during pregnancy. Adherence to all seven food pyramid guidelines was less than $1 \%$ overall, and less than $1 \%$ of participants met all four micronutrient guidelines on vitamin $D$, folate, calcium and iron intake around the time of pregnancy.

Conclusions: Low levels of healthy lifestyle behaviours before pregnancy and low levels of positive lifestyle behaviours during pregnancy demonstrate an urgent need for increased clinical and public health efforts to target deleterious health behaviours before, during and after pregnancy.

\section{INTRODUCTION}

Deleterious health behaviours such as smoking and alcohol consumption are prevalent during the periconceptual window among women in the UK and Ireland. ${ }^{1-3}$ These behaviours persist despite substantial longitudinal evidence of their deleterious offspring health effects, ${ }^{4}{ }^{5}$ clinical trials of interventions to affect behaviour change during pregnancy ${ }^{6-8}$ and widespread policy

\section{Strengths and limitations of this study}

- We provide data on lifestyle behaviours around the time of pregnancy at two time points and for multiple health behaviours.

- However, our data could be influenced by recall biases given its retrospective nature and our dietary data, derived from FFQs do not take account of variation in dietary patterns around the time of pregnancy.

- Despite this, the compatibility of our estimates with other data in the UK and Ireland supports the reliability of the findings.

- Our results highlight important public health concerns regarding the current adequacy of efforts to encourage positive behaviour change before and during pregnancy, and reflect the wider public health issues in the general female population in Ireland.

and practice guidelines for health professionals to target adverse lifestyle exposures. ${ }^{9}$

Up to $70 \%$ of later adult chronic disease shares its risk factors with those targeted in interventions and policy for behavioural change during pregnancy. ${ }^{10}{ }^{11}$ Consequently, as over $80 \%$ of women in high income countries bear at least one child during their reproductive lifetime, ${ }^{12}$ pregnancy provides a single common, widespread opportunity in women's lives to capitalise on increased and sustained contact with health professionals to affect behaviour change that benefits pregnancy outcomes and long-term chronic disease risk. ${ }^{5}{ }^{13}$ Therefore, antenatal care could be used to target adverse offspring health outcomes and reduce women's later chronic disease risk. However, the extent to which positive lifestyle changes are realised during pregnancy is not well documented in Ireland.

In a cross sectional study of 718 women in the South of Ireland, Pregnancy Risk Assessment Monitoring System (PRAMS) Ireland, ${ }^{14}{ }^{15}$ we examined positive lifestyle 
patterns before and during pregnancy in relation to cessation of potentially deleterious health behaviours of smoking and alcohol consumption, and uptake of protective behaviours of dietary changes and folate use.

\section{METHODS}

\section{Sampling strategy}

The details of PRAMS Ireland have been described previously. ${ }^{14}{ }^{15}$ Using hospital discharge records, a sample of women with recent live births was selected from delivery records at Cork University Maternity Hospital, a large urban, obstetric hospital in the South of Ireland, where almost 9000 live-births per year occur (12\% of all Irish births and $66 \%$ of all births in the health services region). ${ }^{16}$ A constant sampling fraction of one in two records alternately sampled 1212 from a sampling frame of approximately 2424 mother-infant pairs discharged between 14 May 2012 and 18 August 2012. Name, address and other demographic and clinical characteristics were recorded. We administered a letter inviting women to participate in the study, three postal surveys, a reminder letter and a telephone follow-up, which included a reminder text. The surveys asked women about their health behaviours and experiences before pregnancy and during pregnancy. A semiquantitative Food Frequency Questionnaire (FFQ) was also administered with the survey asking women to report their usual weekly diet in the 12 months preceding receipt of the questionnaire. Participants were, on average, 4.6 months postdelivery when they completed the questionnaire (minimum 2 months and maximum 9 months). Of the 1212 women sampled, $718(59 \%)$ responded. $^{15}$ Characteristics of non-responders and comparison of responder characteristics with those of the national maternity profile in 2011 have been described in previous publications. ${ }^{14} 15$ In brief, our sample produced a largely representative sample with broadly similar characteristics to the national birth profile, which had a mean age of 32 years, $40 \%$ primiparous women, $33 \%$ single women, $5.8 \%$ preterm birth prevalence, $5.2 \%$ low birth weight prevalence and $28 \%$ caesarean section rate in 2011. ${ }^{17}$ However, responders of PRAMS were older, married and had a higher prevalence of health insurance compared to non-responders which should be taken into account in the interpretation of results.

\section{Statistical analysis and variable definitions}

We examined adherence to major lifestyle guidelines for pregnancy by age, education, ethnicity, body mass index (BMI) and pregnancy intention in Stata V.12 using frequencies and descriptive statistics. For each individual behaviour, all data available irrespective of whether there were missing data for other variables were included resulting in slight variation of sample sizes across different estimates. In particular, we chose this over a complete case analysis, to preserve sample size and retain generalisability where possible, since a complete case analysis would bias our estimates toward women who completed every item of interest to this analysis. However, rates of missing data were generally low and varied from $1.4 \%$ for reporting on alcohol use during pregnancy to $4.6 \%$ for smoking during pregnancy and $0.7 \%$ for demographic variables such as age and education.

Nutritional and dietary variables were defined in line with National Clinical Guidelines on nutrition regarding lifestyle during pregnancy as set out by the Institute of Obstetricians and Gynaecologists in Ireland, Royal College of Physicians of Ireland and the Health Service Executive. $^{18}$ These included intake of a daily supplement of folic acid at least 4 weeks prior to conception and during the first 12 weeks of pregnancy; not smoking before or during pregnancy, not consuming more than 11 units of alcohol per week before pregnancy, not consuming any alcohol during pregnancy (both in line with Irish government guidelines) ${ }^{19}{ }^{20}$ exclusive breastfeeding until 6 months postpartum and engaging in physical activity. Although these guidelines were established after the PRAMS study was conducted, we sought to estimate uptake of positive lifestyle changes prior to the guidelines in order to establish baseline levels of positive behaviour change.

The ages of infants of respondents ranged from 2 to 9 months. Thus, we calculated exclusive breastfeeding up to 2 months postpartum only, given the range of infant ages at which women had responded to the survey. We examined adherence to food pyramid guidelines including intake of six or more servings a day of carbohydrates, five or more servings a day of fruit and vegetables, three servings a day of dairy, two servings a day of cooked meat or protein, two portions of fats and oils, and not more than one serving of foods high in salt, sugar and fat. ${ }^{21}$ We examined micronutrient intake from food for key vitamins and minerals necessary for a healthy pregnancy. This included folic acid intake of $400 \mu \mathrm{g}$ or more per day, vitamin D intake of $10 \mu \mathrm{g}$ or more per day, calcium of $1000 \mathrm{mg}$ or more per day and iron intake of $14 \mathrm{mg}$ or more per day.

Participant's age was derived from discharge data, by subtracting date of birth from maternal date of birth, and categorised into three age groups: 15-29, 30-39 and $40-51$ years. Education was dichotomised to allow those with some third level education (>14 years) to be compared with those having first and second level education only ( $\leq 14$ years). Women's ethnic or cultural background was grouped into either 'White Irish' or 'Other Background' (Other White Background, African, Any Other Black Background, Chinese or Any Other Asian Background). BMI was calculated based on self-reported weight in kilograms $(\mathrm{kg})$ and height in metres $(\mathrm{m})$ at the beginning of pregnancy, and categorised as underweight $\left(<20 \mathrm{~kg} / \mathrm{m}^{2}\right)$, normal $\left(20-25 \mathrm{~kg} / \mathrm{m}^{2}\right)$ and overweight or obese $\left(>25 \mathrm{~kg} / \mathrm{m}^{2}\right)$. Unintended pregnancy was defined as a pregnancy that a woman wanted later (mis-timed) or did not want at any time (unwanted). 


\section{RESULTS}

Of the 718 women participating in the study $(59 \%$ of 1212 women sampled), $23 \%$ were aged $15-29$ years, $71 \%$ were aged $30-39$ years and $5.6 \%$ were aged $40-51$ years. Most women had a third level education $(82.4 \%)$, and most women were White Irish $(80.7 \%)$. Approximately $3.8 \%$ of respondents were underweight, $67 \%$ were normal weight and $29 \%$ were overweight or obese. One-fifth of the women did not intend on being pregnant.

Table 1 describes adherence to health behaviours before and during pregnancy by age, education, ethnicity, BMI and pregnancy intention. Over one quarter of the women $(26.2 \%)$ adhered to all three recommendations on smoking, alcohol and folate use before pregnancy. Lower adherence was evident among younger women $(8.7 \%)$, women with second level education only $(9.6 \%)$, non-Irish women $(11.3 \%)$ and women who did not wish to be pregnant at that time $(6.3 \%)$.

During pregnancy, adherence to guidelines on the same health behaviours increased across all sociodemographic groups to $39 \%$ overall. Differences in adherence reduced due to larger gains in adherence among women with the lowest adherence rates before pregnancy. During pregnancy, 34\% of younger women, 35\% of women with a second level education, $45 \%$ of non-Irish women and $25 \%$ of women who did not wish to be pregnant became adherent to all three smoking, alcohol and folate guidelines. However, although overall adherence to all guidelines increased, gaps in adherence to smoking guidelines during pregnancy remained. For example, a $28 \%$ gap in adherence to smoking guidelines remained between the youngest (15-29 years) and oldest women (40-51 years), despite a narrowing gap between these groups for folate and alcohol use during pregnancy.

Overall, $30 \%$ of women breastfed exclusively up to 2 months postpartum. Lower adherence to this guideline was evident among younger women aged 15-29 years $(21.7 \%)$ vs $32.7 \%$ among women aged $30-39$ years and $30 \%$ among women aged $40-51$ years. Lower adherence was also evident among less educated women (20.8\%) compared with higher educated women $(32.1 \%)$. Irish women $(26.3 \%)$ also had lower adherence levels compared with non-Irish women. Overweight and obese women had lower adherence rates (23\%) compared with underweight and normal weight participants, 32\% and $33 \%$, respectively. However, breastfeeding rates among women who did not intend to be pregnant were similar to those of women who planned their pregnancy $(\sim 30 \%)$.

Table 2 shows adherence to dietary guidelines in the 12 months preceding response to the survey. Less than $1 \%$ of women were adherent to all seven food pyramid guidelines during an average week in the 12 months preceding completion of the questionnaire (a period covering about from about 1 month pre-pregnancy to 2 months post-partum). Only $7.7 \%$ of women reached the

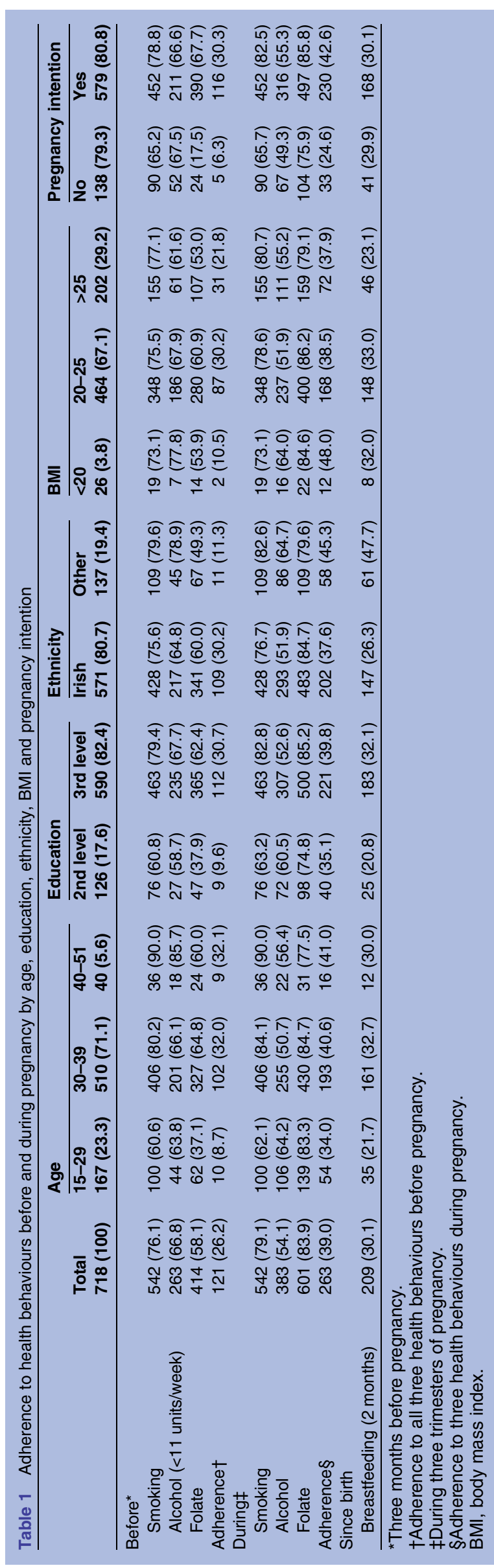




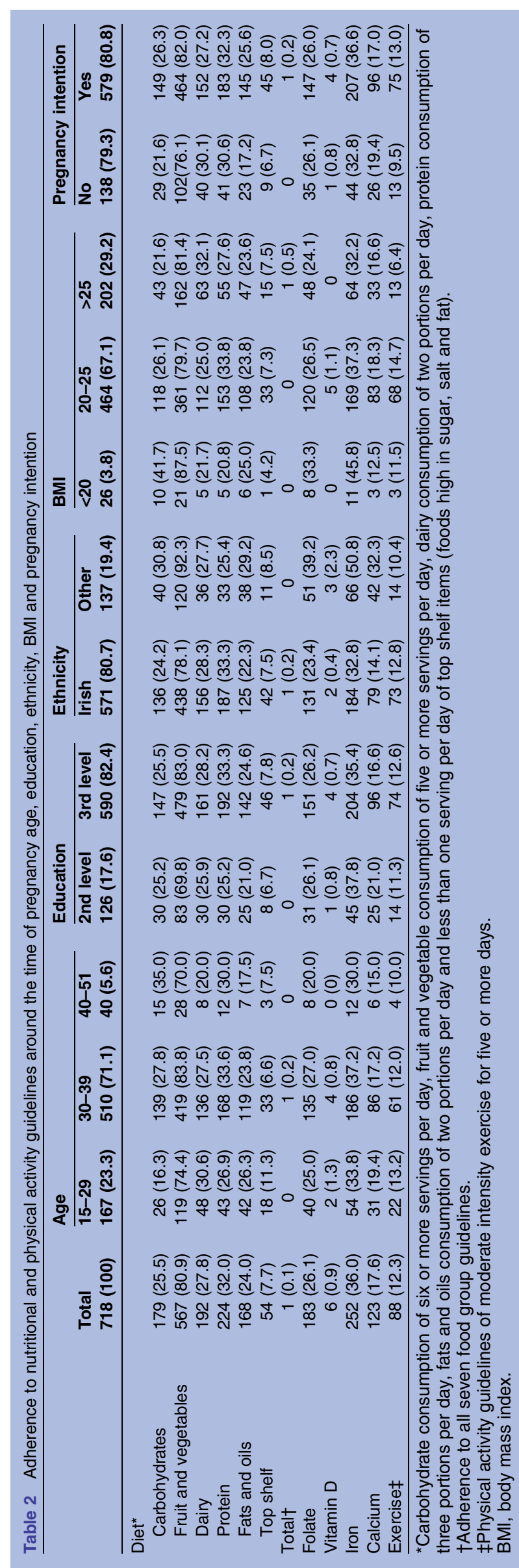

recommended guideline of consumption of less than one serving per day of foods high in salt, fat and sugar. However, $81 \%$ of women reported meeting fruit and vegetable consumption guidelines of five or more servings per day. For carbohydrate, dairy, protein and oils intake, $24-32 \%$ of women met the guidelines. Similarly, adherence to physical activity guidelines of moderate intensity activity on five or more days of the week was low overall, at $12.3 \%$. In general, adherence to dietary and physical activity guidelines was low across all age groups and did not appear to substantially differ by age, education, ethnicity, BMI and pregnancy intention.

Micronutrient analysis revealed that, regarding some of the key nutrients necessary for a healthy pregnancy, only $26 \%$ of women reached folate recommendations, $0.9 \%$ reached vitamin $\mathrm{D}$ recommendations, $36 \%$ met calcium recommendations and $17.6 \%$ met iron recommendations, from food. Major differences in micronutrient intake by age and education were not observed. However, non-Irish women appeared to have higher adherence to all micronutrient intakes, compared with Irish women.

\section{DISCUSSION}

In this cross sectional study of 718 women in the South of Ireland, we found a low prevalence of positive lifestyle behaviours before pregnancy $(26 \%)$, which changed to $39 \%$ in early pregnancy. Furthermore, we found less than $1 \%$ of women were meeting nutritional or micronutrient guidelines around the time of pregnancy. Critically, we found that low adherence was prevalent across all social groups but was even more pronounced among younger and less educated women.

\section{IMPLICATIONS}

Up to $70 \%$ of most chronic diseases may be prevented through early intervention and lifestyle modification including smoking cessation, engaging in regular physical activity, and consuming a healthy and varied diet with moderate alcohol consumption. ${ }^{11}{ }^{22}$ These behaviours have been the focus of many of Ireland's longterm policy and public health initiatives, ${ }^{23}{ }^{24}$ and, more recently, also of 'Healthy Ireland', Ireland's newest framework focusing on behaviour change and wellbeing in the population. ${ }^{25}$ Consequently, our finding that only $26 \%$ of women are adherent to all lifestyle behaviours in advance of pregnancy has important implications for public health initiatives at large, as these lifestyle patterns reflect the wider lifestyle patterns of women in the Irish population. For example, although a workplace smoking ban has existed in Ireland since $2004,{ }^{26}$ and smoking rates in the general population have declined by about $8 \%,{ }^{27}$ the relatively high rates of smoking prior to and during pregnancy in PRAMS suggest that further efforts to support smoking cessation in women in the general population and before they become pregnant are required. In relation to breastfeeding, although the 
Maternity Protection (Amendment) act was introduced in $2004^{28}$ to allow women in employment to take time off work each day to breastfeed, employers are currently not obliged to provide workplace facilities to do so. Therefore, an examination of more comprehensive workplace initiatives and legislation to encourage breastfeeding may be beneficial. In addition, considering the role of primary care and women's contact with health providers or health promotion before pregnancy in order to establish a continuum of behavioural counselling focusing on improving women's lifestyle behaviours generally, would have benefits for health behaviours prior to and during pregnancy, and for behaviour change efforts from first booking visit through antenatal care. In relation to maternity care specifically, maternity care is predominantly hospital based and consultant-led in Ireland $(0.2 \%$ of births occurring at home $),{ }^{29}$ while postnatal follow-up is carried out by the public health nursing service, with at least one home visit usually 2 davs after birth and a follow-up check-up provided at 6 weeks by the general practitioner. ${ }^{30}$ Therefore, examining how current primary care, antenatal and postnatal care services could develop a more integrated approach to supporting behaviour change in women is required. However, given the low level of positive lifestyle behaviours in PRAMS participants prior to pregnancy, our findings reflect a wider public health problem that requires an upscaling of current public health efforts in the general population. Moreover, a system for monitoring how lifestyle patterns change over time including those of pregnancy at the national and regional level may be required so that the effects of new public health efforts on health behaviour change can be monitored.

While, in the general population, wider public health initiatives are required, the findings also have some implications for initiatives and care within and after pregnancy. For example, in the 2005 government strategic action plan on breastfeeding, ${ }^{31}$ two specific 5-year targets included development of a comprehensive, accurate and timely infant feeding data collection system within 5 years of the plan and achievement of $100 \%$ baby-friendly hospital status; however, currently, only 9 of 20 Irish maternity hospitals have been assessed as achieving baby-friendly hospital status-the WHO/UNICEF quality initiative for encouraging breastfeeding, which includes providing staff training, policy and support groups. ${ }^{32}$ Moreover, a comprehensive infant feeding data collection system has not yet been establishedmore than 10 years after the action plan was written. Regarding antenatal counselling, standard care during pregnancy currently includes assessment for alcohol, smoking and substance abuse, with subsequent referral to the national smoking cessation programme and discussion with a consultant on alcohol or substance abuse, when necessary. However, in relation to dietary and exercise guidelines, only high risk groups receive dietary advice, and our data showing that less than $1 \%$ of women were adhering to all nutrition guidelines during pregnancy suggest that this should be established for all women. In addition, providing greater depth and scope to the 2013 'Nutrition in Pregnancy'18 guidelines for care providers may be beneficial, including extensive coverage on alcohol use during pregnancy, which is currently lacking. More specific and detailed training, key performance indicators for care providers and incentives for all healthcare providers to counsel women on their behaviours may be beneficial. Innovation in health education approaches may also be useful-including the use of social media and other non-conventional routes to influence lifestyle change.

\section{Strengths and limitations}

There are a number of strengths to this study, (1) including collection of data on a wide variety of important health behaviours and experiences, (2) detailed nutritional data around the time of pregnancy and (3) data on behaviours for two phases: before and during pregnancy. However, recall bias is a substantial threat to the validity of our results, particularly for self-reported height and weight, and socially unacceptable lifestyle behaviours such as alcohol consumption and smoking. Second, FFQ's do not take account of variation in dietary patterns that may occur between the prepregnancy, antenatal and postnatal period. However, the high level of comparability of our data with those from other similar populations within the UK and Ireland ${ }^{33} 34$ provides reassurance that our findings are valid and generalisable.

\section{CONCLUSION}

We observed low levels of protective lifestyle behaviours before pregnancy and limited increases in positive behaviours during pregnancy, demonstrating a need for increased public health and clinical efforts to target deleterious health behaviours both in the general population and during pregnancy. Given that these behavioural targets are also key contributors to later adult chronic disease, further research, interventions and policy that focus on developing supportive environments for behaviour change in the Irish population are required.

\section{Author affiliations}

${ }^{1} \mathrm{MRC}$ Integrative Epidemiology Unit at the University of Bristol, School of Social and Community Medicine, Bristol, UK

${ }^{2}$ Department of Epidemiology and Public Health, University College Cork, Cork, Ireland

${ }^{3} \mathrm{HRB}$ Clinical Research Facility Cork, Mercy University Hospital, Cork, Ireland ${ }^{4}$ School of Medicine, University College Cork, Cork, Ireland

${ }^{5}$ National Perinatal Epidemiology Centre, Cork University Maternity Hospital, Cork, Ireland

Twitter Follow Linda O'Keeffe @LindaM_OKeeffe Darren Dahly @statsepi, Janas Harrington @janasharrington, Richard Greene @dickgreene5 and Patricia Kearney @TrishCork

Contributors LMOK had the original idea for the study, wrote the first draft of the article, applied critical revisions to the article based on other coauthor recommendations and approved the final version for publication. PMK, PC 
and RAG conceptualised the design of the study, assisted in interpretation of the data, reviewed and revised the manuscript and approved the final version for publication. MM contributed to the critical revisions of the article and approved the final version for publication. DLD and JMH assisted in the interpretation of the data, reviewed and revised the manuscript and approved the final version for publication.

Funding PRAMS was conducted with the administrative and financial support of the National Perinatal Epidemiology Centre, Cork, as well as with staff of Cork University Maternity Hospital, and with assistance from the Health Research Board (HRB) in Ireland under Grant Number PHD/2007/16. LMOK is supported by a UK Medical Research Council Population Health Scientist fellowship (MR/M014509/1).

Competing interests None declared.

Ethics approval This research protocol and all study materials received ethical approval from the Clinical Research Ethics Committee of the Cork Teaching Hospitals.

Provenance and peer review Not commissioned; externally peer reviewed.

Data sharing statement No additional data are available.

Open Access This is an Open Access article distributed in accordance with the Creative Commons Attribution Non Commercial (CC BY-NC 4.0) license, which permits others to distribute, remix, adapt, build upon this work noncommercially, and license their derivative works on different terms, provided the original work is properly cited and the use is non-commercial. See: http:// creativecommons.org/licenses/by-nc/4.0/

\section{REFERENCES}

1. O'Keeffe LM, Kearney PM, McCarthy FP, et al. Prevalence and predictors of alcohol use during pregnancy: findings from international multicentre cohort studies. BMJ Open 2015;5:e006323.

2. McCarthy FP, O'Keeffe LM, Khashan AS, et al. Association between maternal alcohol consumption in early pregnancy and pregnancy outcomes. Obstet Gynecol 2013;122:830-7.

3. O'Keeffe LM, Kearney PM, Greene RA, et al. Maternal alcohol use during pregnancy and offspring trajectories of height and weight: a prospective cohort study. Drug Alcohol Depend 2015;153:323-9.

4. Patra J, Bakker $\mathrm{R}$, Irving $\mathrm{H}$, et al. Dose-response relationship between alcohol consumption before and during pregnancy and the risks of low birthweight, preterm birth and small for gestational age (SGA)-a systematic review and meta-analyses. BJOG 2011;118:1411-21.

5. McCowan LM, Dekker GA, Chan E, et al. Spontaneous preterm birth and small for gestational age infants in women who stop smoking early in pregnancy: prospective cohort study. BMJ 2009;338:b1081.

6. Coleman T, Chamberlain C, Davey MA, et al. Pharmacological interventions for promoting smoking cessation during pregnancy. Cochrane Database Syst Rev 2012;9:CD010078.

7. Stade BC, Bailey C, Dzendoletas D, et al. Psychological and/or educational interventions for reducing alcohol consumption in pregnant women and women planning pregnancy. Cochrane Database Syst Rev 2009;(2):CD004228.

8. Chamberlain C, O'Mara-Eves A, Oliver S, et al. Psychosocial interventions for supporting women to stop smoking in pregnancy. Cochrane Database Syst Rev 2013;10:CD001055.

9. National Institute for Health and Care Excellence. Antenatal care: routine care for healthy pregnant women: NICE clinical guidelines 62. 2008.

10. Tábak AG, Herder C, Rathmann W, et al. Prediabetes: a high-risk state for diabetes development. Lancet 2012;379:2279-90.
11. Capewell S, Allender S, Critchley J, et al. Modelling the UK burden of cardiovascular disease to 2020. London: Cardio \& Vascular Coalition and the British Heart Foundation, 2009.

12. Martinez G, Daniels K, Chandra A. Fertility of men and women aged 15-44 years in the United States: National Survey of Family Growth, 2006-2010. Natl Health Stat Rep 2012;51:1-28.

13. Di Angelantonio E, Kaptoge S, Wormser D, et al. Association of cardiometabolic multimorbidity with mortality. JAMA 2015;314:52-60.

14. O'Keeffe LM, Kearney PM, Greene RA. Surveillance during pregnancy: methods and response rates to a hospital based cross sectional study of the Pregnancy Risk Assessment Monitoring System in Ireland. BMC Pregnancy Childbirth 2013;13:180.

15. O'Keeffe LM, Kearney PM, Greene RA. Pregnancy risk assessment monitoring system in Ireland: methods and response rates. Matern Child Health J 2015;19:480-6.

16. Cork University Maternity Hospital. Cork University Maternity Hospital Annual Report 2010. Cork, 2010.

17. Economic and Social Research Institute. Perinatal statistics report 2011. Dublin, Ireland: Health Research and Information Division, 2012.

18. Health Services Executive Ireland. Clinical practice guideline: nutrition for pregnancy: guideline No.27. 2013. https://www.hse.ie/ eng/about/Who/clinical/natclinprog/obsandgynaeprogramme/nutpreg pdf

19. Health Services Executive Ireland. Alcohol and pregnancy 2009 [updated 200910/01/2014]. http://www.yourdrinking.ie/ alcohol-and-pregnancy

20. Health Services Executive Ireland. Alcohol 2013. http://www.hse.ie/ go/alcohol/

21. Food Safety Authority of Ireland. Healthy eating and active living for adults, teenagers and children over 5 years: a food guide for health professionals and catering services. Dublin: Food Safety Authority of Ireland, 2011.

22. Ford ES, Capewell S. Proportion of the decline in cardiovascular mortality disease due to prevention versus treatment: public health versus clinical care. Annu Rev Public Health 2011;32:5-22.

23. Department of Health. National alcohol policy. Dublin: Stationary Office, 1996.

24. Department of Health. Tobacco free Ireland. Dublin: Stationary Office, 2013

25. Department of Health. Healthy Ireland - a framework for improved health and wellbeing 2013-2025. Dublin, 2013.

26. Government of Ireland. Tobacco Smoking (Prohibition) Regulations 2003. 2003. http://www.irishstatutebook.ie/eli/2003/si/481/made/en/ print

27. Hickey P, Evans D. Smoking in Ireland 2014: synopsis of key patterns. Dublin: Health Services Executive, 2015.

28. Government of Ireland. Maternity Protection (Amendment) Act 2004. 2004. http://www.irishstatutebook.ie/eli/2004/act/28/enacted/en/html

29. Meaney S, Sweeney S, Deaney J, et al. Planned home births in Ireland annual report 2012; HSE National Home Birth Service provided by Self Employed Community Midwives. Cork: Health Services Executive, 2013.

30. Begley C, Devane D, Clarke M, et al. Comparison of midwife-led and consultant-led care of healthy women at low risk of childbirth complications in the Republic of Ireland: a randomised trial. BMC Pregnancy Childbirth 2011;11:85.

31. Nic Gabhainn S, Batt V. Breastfeeding in Ireland: a 5 year strategic action plan. In: Department of Health and Children, ed. Dublin: Health Services Executite, 2005.

32. Baby Friendly Hospital Initiative in Ireland. What is the Baby Friendly Hospital Initiative in Ireland? 2015. http://www.babyfriendly.ie

33. Kelly $\mathrm{Y}$, Sacker A, Gray R, et al. Light drinking in pregnancy, a risk for behavioural problems and cognitive deficits at 3 years of age? Int $J$ Epidemiol 2009;38:129-40.

34. Ray JG, Singh G, Burrows RF. Evidence for suboptimal use of periconceptional folic acid supplements globally. BJOG 2004;111:399-408. 